\title{
The Boundary Layer Flows of a Rivlin-Ericksen Fluid
}

\author{
K. Sadeghy, N. Khabazi*, S. M. Taghavi
}

University of Tehran, Department of mechanical Eng., North Kargar St., Jalale aleahmad Blvd.. Tehran, Iran

Email: nkhabazi@ut.ac.ir

\begin{abstract}
The present work deals with the two-dimensional incompressible, laminar, steady-state boundary layer equations. First, we determine a family of velocity distributions outside the boundary layer such that these problems may have similarity solutions. We study the Falkner-Skan flow of a viscoelastic fluid governed by second order model, as the Reynolds number $R e \rightarrow \infty$. We obtain an ordinary forth order differential equation to obtain the stream function, velocity profile and the stress. The stream function is then governed by a generalized Falkner-Skan equation. In comparison with Newtonian Falkner Skan equation that has two coefficients this new one has four coefficients that two of them represent elastic properties of the fluid. The effects of the elastic parameter on the velocity filed have been discussed. As it is shown in the figure there is a good agreement between numerical results and previous special cases confirm the validity of the presented algorithm.
\end{abstract}

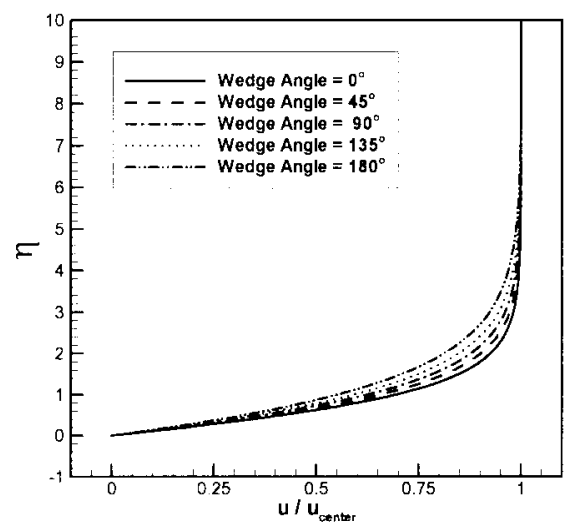

Figure: Velocity profiles for wedge flow with $D e=1$

\section{REFERENCES}

1. Rivlin RS, Ericksen JL. Stress deformation relation for isotropic material. J. Rational Mech. Anal., $1955 ; 4: 323-425$

2. Rajagopal KR. A note on unsteady unidirectional flows of a non-Newtonian fluid. Int. J. NonLinear Mech., 1982; 17: 369-373 